\title{
FACE RECOGNITION USING DIFFERENT LOCAL FEATURES WITH DIFFERENT DISTANCE TECHNIQUES
}

\author{
M.Koteswara Rao ${ }^{1}$, K.Veera Swamy ${ }^{2}$, K.Anithasheela ${ }^{3}$ and B.Chandra Mohan ${ }^{4}$ \\ ${ }^{1}$ Associate Professor, QIS College of Engineering \& Technology, Ongole, A.P, India, \\ koteshprojectegmail.com \\ ${ }^{2}$ Professor, QIS College of Engineering \& Technology, Ongole, A.P, India, \\ kilarivs@yahoo.com \\ ${ }^{3}$ Associate Professor, JNTUH, Hyderabad, A.P, India, \\ kanithasheela@gmail.com \\ ${ }^{4}$ Professor, Bapatla Engineering College, A.P, India, \\ chandrabhuma@gmail. com
}

\begin{abstract}
A face recognition system using different local features with different distance measures is proposed in this paper. Proposed method is fast and gives accurate detection. Feature vector is based on Eigen values, Eigen vectors, and diagonal vectors of sub images. Images are partitioned into sub images to detect local features. Sub partitions are rearranged into vertically and horizontally matrices. Eigen values, Eigenvector and diagonal vectors are computed for these matrices. Global feature vector is generated for face recognition. Experiments are performed on benchmark face YALE database. Results indicate that the proposed method gives better recognition performance in terms of average recognized rate and retrieval time compared to the existing methods.
\end{abstract}

\section{KEYWORDS}

Vertical centered, Horizontal centered, Eigen vector, Eigen values and distance methods.

\section{INTRODUCTION}

Face recognition [1] is a computer application for identifying and verifying a person from the face database. The face recognition system is generally used in security purpose and can be compared to other techniques such as fingerprint, iris and signature. More face recognition methods are identify face extract feature, or from an image of the database. These methods may analyze the relative size, shape, position of the database images. These extracted features [5] are used to search for other relevant images. So far face recognition methods developed can be classified as holistic method or local feature method. First method is appearance based technique, which analyze the distribution of individual faces in face space for holistic features. It can be done by using global and local features[4]. Concentration on dynamic link matching or graph matching is considered in local feature [1] method. In global feature method or holistic method concentration on eigenfaces or similar appearance such as Principal Component Analysis (PCA) is considered. PCA approach is mainly concentrated on dimensionality reduction. This scheme is based on linearly projecting the image space to a lower dimensionality space that is also known as Eigen space. Second method is feature based technique [5], which is concentrated on dimensionality of input image as well as images in face database. In face recognition system dimensionality reduction [7] is an essential technique. Block diagram of face recognition system is shown in Figure 1 .

DOI : 10.5121/ijcseit.2012.2107 


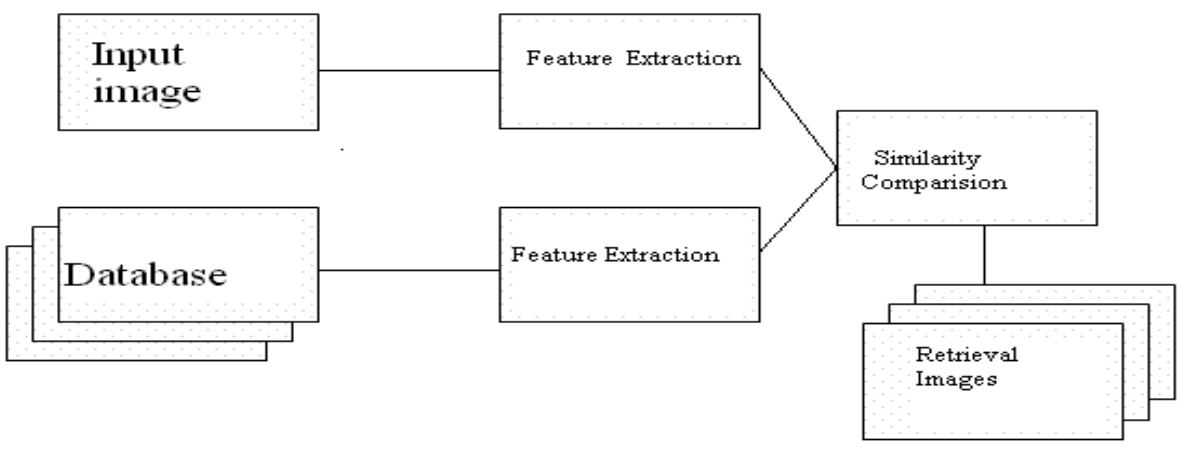

Figure 1. Block diagram of face recognition.

\section{DISTANCE MEASURES}

Several distance measures are useful for face recognition. Manhattan distance, weighted angle based Distance, and Minkowski Distance are considered in this work.

\subsection{Manhattan Distance}

Manhattan distance, also known as the $\mathrm{L}_{1}$-distance, between two points in an Euclidean space fixed Cartesian coordinate system sum of the lengths of the projections of the line segment between the points onto the coordinate axes. Notice that the Manhattan distance depends on the choice on the rotation of the coordinate system, but does not depend on the translation of the coordinate system or its reflection with respect to a coordinate axis. Manhattan distance is also known as city block distance.

$$
d(Y, Z)=L_{p_{=1}}(Y, Z)=\sum_{i=1}^{n}\left|x_{i}-y_{i}\right|
$$

Where $\mathrm{n}$ is the number of variables, and $\boldsymbol{X} i$ and $\boldsymbol{Y} i$ are the values of the $i$ th variable, at points $\mathrm{Y}$ and $\mathrm{Z}$ respectively.

\subsection{Weighted angle Distance}

Cosine similarity [4] is a measure of similarity between two vectors by measuring the cosine of the angle between them. The cosine of 0 is 1 , and less than 1 for any other angle; the lowest value of the cosine is -1 . The cosine of the angle between two vectors thus determines whether two vectors are pointing in roughly the same direction. Cosine of two vector $\mathrm{s}$ can be written as

$$
\text { Similarity }=\cos (\theta)=\frac{Q . Y}{\|Q\| .\|Y\|}=\sum Q_{I} * Y_{I} \frac{\sum_{I=1}^{N} Q_{I} * Y_{I}}{\sqrt{\sum_{I=1}^{N}\left(Q_{I}\right)^{2}} * \sqrt{\sum_{I=1}^{N}\left(Y_{I}\right)^{2}}}
$$

The resulting similarity ranges from -1 meaning exactly opposite, to 1 meaning exactly the same, with 0 usually indicating independence, and in-between values indicating intermediate similarity or dissimilarity. For text matching, the attribute vectors $A$ and $B$ are usually the term frequency 
vectors of the documents. The cosine similarity can be seen as a method of normalizing document length during comparison.

\subsection{Minkowski Distance}

Minkowski distance is concentrated on Euclidean [4] space, which can be considered as a generalization of both Euclidean and Manhattan distance for getting more recognition efficiency. Minkowski distance is based on factor $\mathrm{p}$.

$$
p=\left(x_{1}, x_{2}, \ldots . x_{n}\right)
$$

Minkowski diastance is typically used with $\mathrm{p}$ being 1 or 2 . In the limiting case of $\mathrm{p}$ reaching infinity we obtain the chebyshev distance.

$$
d(Y, Z)=L_{p}(Y, Z)=\left(\sum_{i=1}^{n}\left|x_{i}-y_{i}\right|^{p}\right)^{i / p}
$$

Minkowski distance is often used when variables are measured on ration scales with absolute zero value. Variables with a wider range can overpower the result.

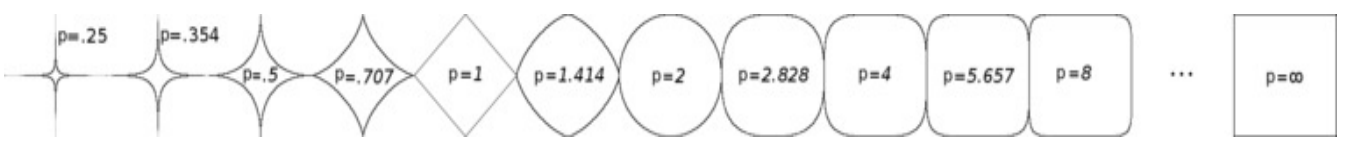

Figure 2 Minkowski distance

\section{PROPOSED ALGORITHM}

Proposed algorithm to get feature vector for the database is given below:

1. Each image is partitioned into sub images. $D=r 1 * r 2 / k . r 1 \& r 2$ are the image rows $\&$ columns. $\mathrm{K}$ is the equally sized.

2. Convert the each sub image into column data matrix. Each of them can be expressed in the order of a D-by-N. $\mathrm{C}_{\mathrm{i}}=\left\{\mathrm{c}_{\mathrm{i} 1}+\mathrm{c}_{\mathrm{i} 2}+\mathrm{c}_{\mathrm{i} 3}+\ldots \mathrm{c}_{\mathrm{iN}}\right\}$ with $\mathrm{i}=1,2, \ldots \ldots . \mathrm{K}$. here $\mathrm{N}$ is the total number of images

3. Calculate mean value for each sub image.

4. Subtract the mean value from column data matrix of each sub image then obtain vertically centered column data matrix $\mathrm{C}_{\mathrm{vi}}=\left\{\hat{\mathrm{c}}_{\mathrm{i} 1}+\hat{\mathrm{c}}_{\mathrm{i} 2}+\hat{\mathrm{c}}_{\mathrm{i} 3}+\ldots \hat{\mathrm{c}}_{\mathrm{iN}}\right\}$ with $\mathrm{i}=1,2, \ldots \ldots \ldots \mathrm{K}$.

5. Rearrange the elements to get square matrix.

6. Collect Eigen values, Eigen vectors, and diagonal values of the square matrix $P_{V i}=\left\{P_{i 1}+P_{i 2}\right.$ $\left.+\mathrm{P}_{\mathrm{i} 3}+\ldots \ldots+\mathrm{P}_{\mathrm{iL}}\right\}$ with $\mathrm{i}=1,2 \ldots . \mathrm{S}$. here $\mathrm{L}$ is the feature of the sub image. Then obtain training data base matrix $\mathrm{G}_{\mathrm{vi}}=\mathrm{P}_{\mathrm{vi}_{\mathrm{i}}^{\mathrm{T}}}^{\mathrm{T}} \mathrm{C}_{\mathrm{vi}}=\left\{\mathrm{G}_{\mathrm{i} 1}+\mathrm{G}_{\mathrm{i} 2}+\ldots \ldots+\mathrm{G}_{\mathrm{iN}\}}=1,2 \ldots \mathrm{K}\right.$.

7. Repeat the same procedure row data matrix.

8. Reduce the feature size as per the requirement. $G_{v j}=\left(G_{1 j}{ }^{T}, G_{2 J}{ }^{T}, \ldots \ldots G_{S j}{ }^{T}\right)^{T}, j=1,2, . . N$.

9. Convert the feature into global feature by converting into a single value. 
Proposed algorithm to retrieve the related face images:

1. Repeat the same procedure as explained above for the query image.

2. Apply different distance measures to retrieve the relevant faces from the database.

3. Display the relevant faces based on minimum distance criteria.

\section{EXPERIMENTAL RESULTS}

Recognition performance in terms of average recognition rate and recognition time of the proposed face recognition system is tested by conducting experiments on yale data base. A face database ${ }^{[6]}$ test set was constructed by selecting 100 images of 10 individuals, ten images per person. These images of a person used for training and testing. the experimental results are tabulated in Table 1. Since the recognition accuracy of the sub-pattern image, several sizes of sub-pattern images are used in our experiments as shown below: $56 \times 46(\mathrm{~S}=4), 28 \times 23(\mathrm{~S}=16)$, $14 \times 23(\mathrm{~S}=32), 7 \times 23(\mathrm{~S}=64)$, and $4 \times 23(\mathrm{~S}=112)$. Results have been presented in two models. First model is named as hybrid approach1. In this, vertical, horizontal, and whitened are considered. Second model is named as hybrid approach2. In this, eigen values, vertical, horizontal, and whitened are considered.

\subsection{Feature selection}

Feature selection procedure is shown in Figure 3.

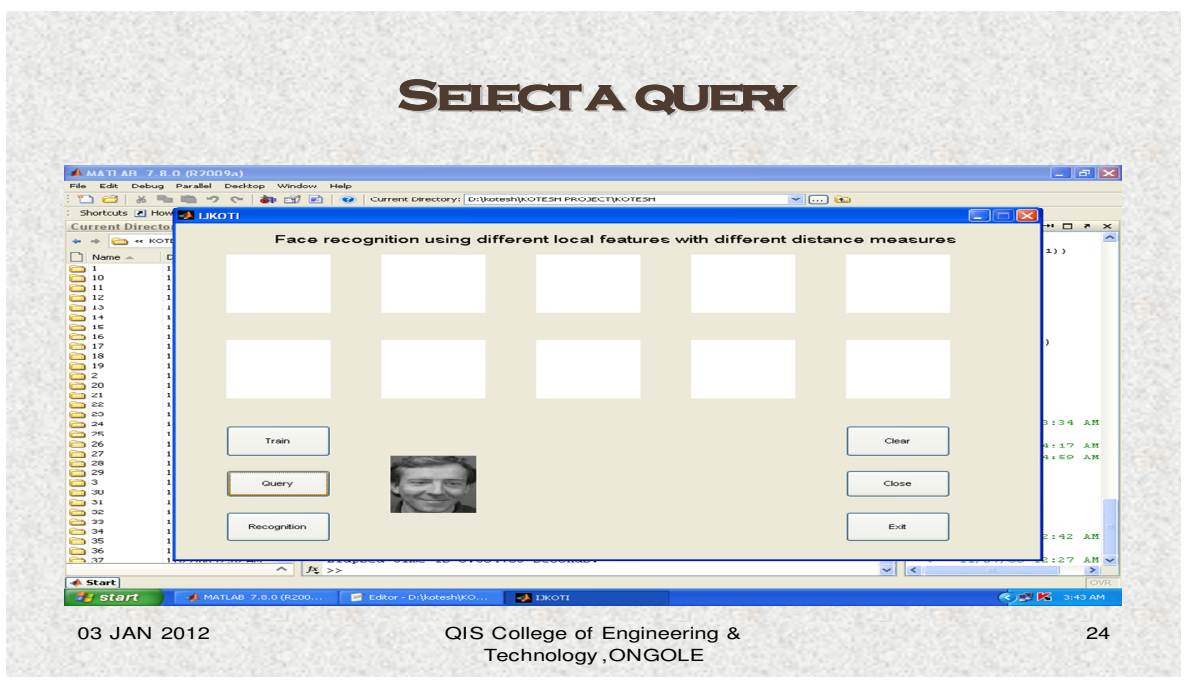

Figure 3. Query Image

From the query image feature is taken based on sub-pattern method. In this paper we take only 64 feature of this query image. That may be depends up on the sub-parts of this image $(S=16)$. For each sub-pattern we consider four positive eigenvectors that is largest eigenvector of the sub-part. Comparative performance of all training global feature with this query image finally recognized results images with top left image as query image. 


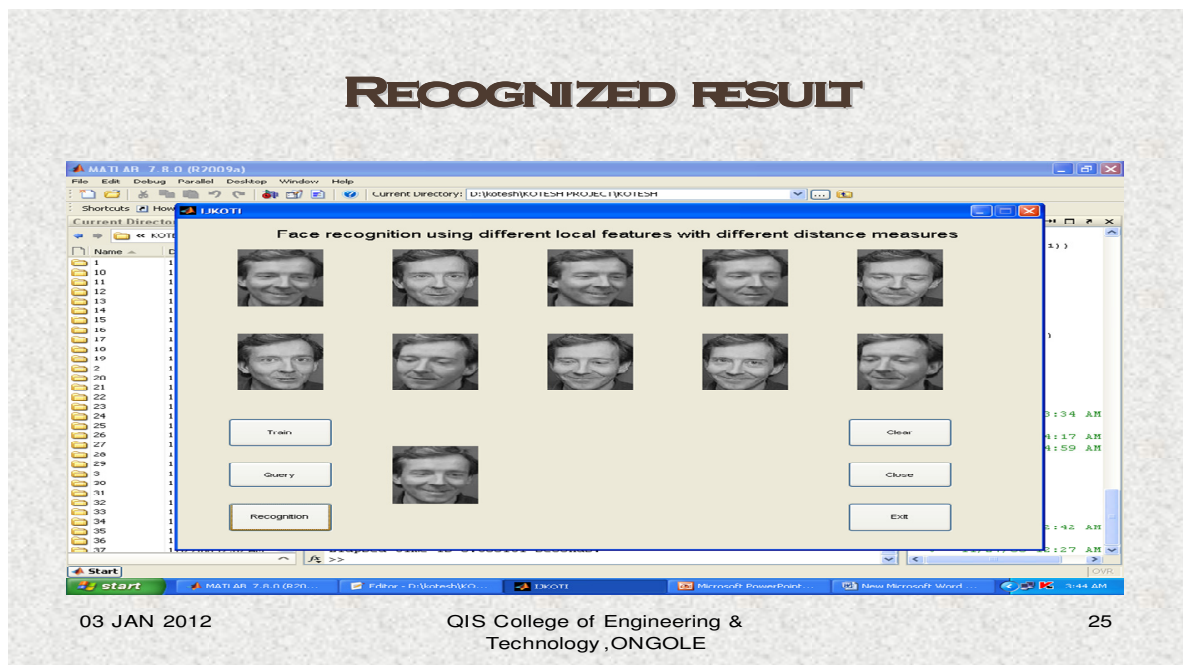

Figure 4. Recognized result

\subsection{Recognized rate}

Comparative performance in terms of average recognized rate is shown in Figure 5 and Figure 6 respectively

\subsubsection{Hybrid approach1 experimental results}

Table 1. Recognized efficiency on face database for hybrid approach1.

\begin{tabular}{|c|l|c|c|c|c|c|}
\hline Feature Technique & $\begin{array}{c}\text { Distance } \\
\text { Measure }\end{array}$ & 1 & 3 & 5 & 7 & 10 \\
\hline & $\begin{array}{l}\text { Manhattan } \\
\text { Distance }\end{array}$ & 100 & 50 & 41 & 37.85 & 35.75 \\
\cline { 2 - 7 } $\begin{array}{c}\text { Hybrid approach1 } \\
\text { (Vertical+Horizontal+ } \\
\text { Whitened) }\end{array}$ & $\begin{array}{l}\text { Weighted } \\
\text { angle } \\
\text { Distance }\end{array}$ & 100 & 99.16 & 96.5 & 87.85 & 79.25 \\
\cline { 2 - 7 } & $\begin{array}{l}\text { Minkowski } \\
\text { Distance }\end{array}$ & 100 & 99.16 & 95 & 88.57 & 80.75 \\
\hline
\end{tabular}


International Journal of Computer Science, Engineering and Information Technology (IJCSEIT), Vol.2, No.1, February 2012

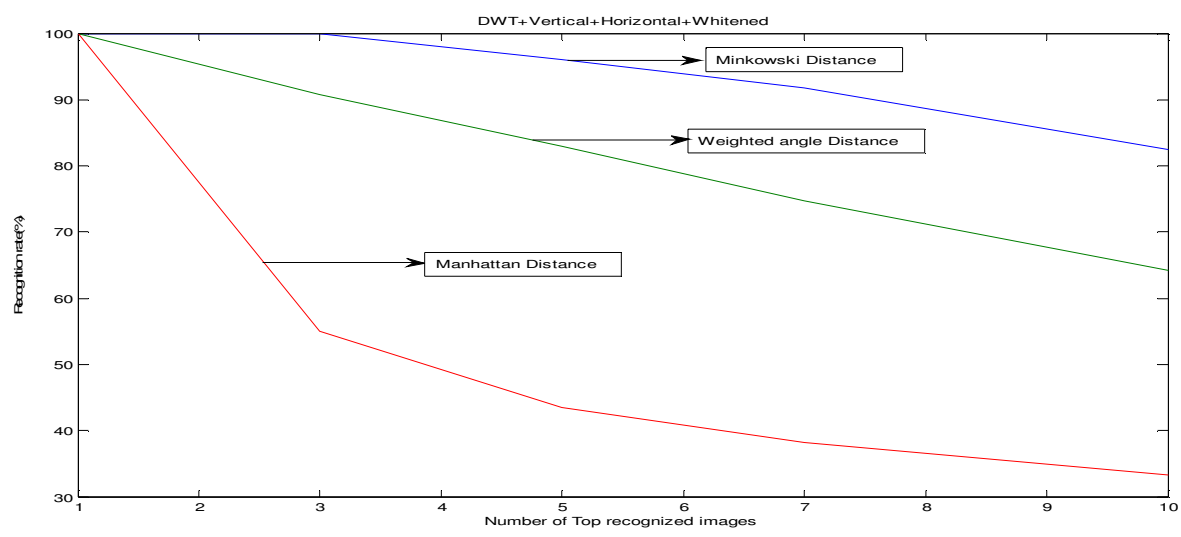

Figure 5. Comparative recognition rates for Hybrid approach1

\subsubsection{Hybrid approach2 experimental results}

Table 2. Recognized efficiency on face database for hybrid approach 2.

\begin{tabular}{|c|l|c|c|c|c|c|}
\hline Feature Technique & $\begin{array}{c}\text { Distance } \\
\text { Measure }\end{array}$ & 1 & 3 & 5 & 7 & 10 \\
\hline & $\begin{array}{l}\text { Manhattan } \\
\text { Distance }\end{array}$ & 100 & 53.3 & 43.50 & 36.78 & 33.25 \\
\cline { 2 - 7 } $\begin{array}{c}\text { Hybrid approach2 } \\
\text { (Eigenvalue + Vertical+ } \\
\text { Horizontal+Whitened) } \\
\text { (PROPOSED) }\end{array}$ & $\begin{array}{l}\text { Weighted } \\
\text { angle } \\
\text { Distance }\end{array}$ & 100 & 95.83 & 88 & 86.78 & 74.25 \\
\cline { 2 - 7 } & $\begin{array}{l}\text { Minkowski } \\
\text { Distance }\end{array}$ & $\mathbf{1 0 0}$ & $\mathbf{1 0 0}$ & $\mathbf{9 6}$ & $\mathbf{9 0}$ & $\mathbf{8 2}$ \\
\hline
\end{tabular}




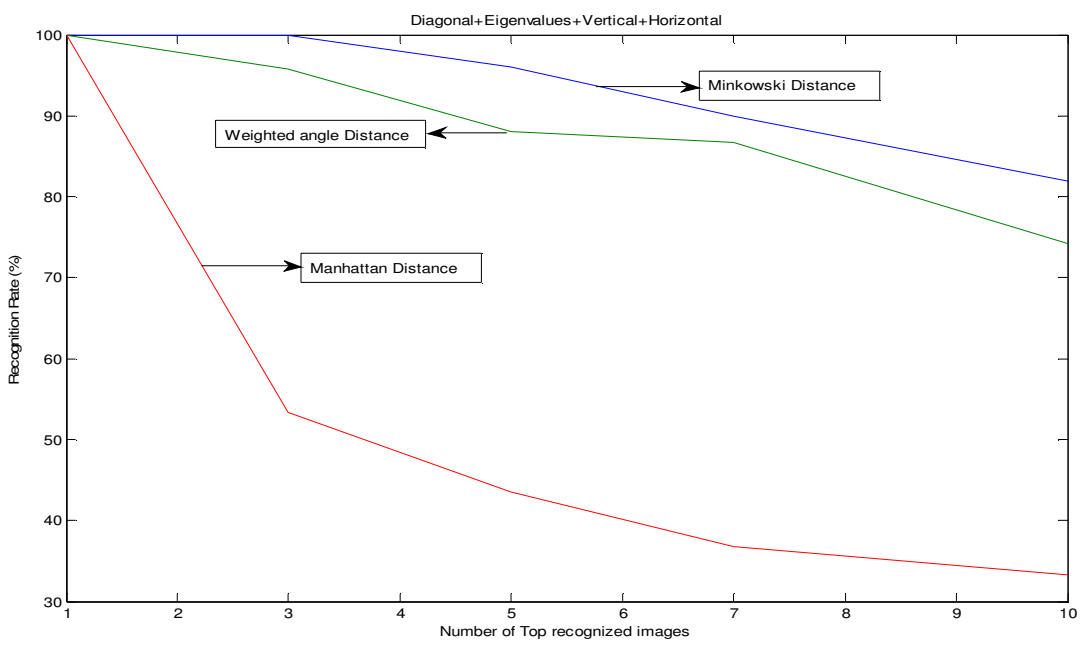

Figure 6. Comparative recognition rates for Hybrid approach2

Results are indicating that Hybrid approach2 give better result with Minkowski distance compared to hybrid approach1 with all distance measures.

\subsection{Recognition Time}

Face recognition using different local features with different distance measures is presented. Time taken for generating the features for the entire database is 52.98 seconds for hybrid approach 1 . Recognition time for Minkowski, Weighted angle and Manhattan are 0.46, 0.45, 0.43seconds respectively. Similarly database time for hybrid approach2 is 51.84 seconds. Recognized time for Minkowski, Weighted angle and Manhattan are $0.45,0.42,0.38$ seconds respectively.

\section{CONCLUSIONS}

Face recognition using different local features with different distance measures are presented in this paper. Global feature vectors are generated based on eigenvalues, eigenvectors and diagonal values with whitened features. Local features are based on vertical and horizontal sub pattern techniques. Proposed method with Minkowski distance gives better results in term of average recognized rate and retrieval time compared to the existing methods.

\section{ACKNOWLEDGEMENTS}

Authors would like to thank the Dr.S.Srinivas Kumar, Director R\&D Cell, JNTUK, Kakinada for his valuable thoughts. Authors thank to the Dr.Ch.Srinivasa Rao, Sai Aditya Engineering College, surampalem for his valuable discussions related to this work.

\section{REFERENCES}

[1] W.Arnold. M. Smeulders, M. Worring, S. Satini A. Gupta, R. Jain. Content - Based Image Retrieval at the end of the Early Years, IEEE Transactions on Pattern analysis and Machine Intelligence, Vol. 22, No:12, pp 1349-1380, 2000.

[2] Gupta. Visual Information Retrieval Technology: A Virage erspective, Virage Image Engine. API Specification, 1997.

[3] Ping-Cheng, Pi-Cheng Tung, A Novel Hybrid Approach Based On Subpattern Technique and Whitened PCA for Face Recognition, Pattern Recognition 42 (2009), 978-984. 
[4] Vytautas Perlibakas Distance measures for PCA based face recognition, Pattern Recognition letters 25 (2004), 711-724

[5] S.I. Choi, Kim, C.H. Choi, Shadow compensation in 2D images for face recognition, Pattern Recognition 40 (2007) 2118-2125.

[6] M. Turk, Pentland, Eigenfaces for recognition, J. Cognitive Neurosci. 3 (1) (1991) 71-86.

[7] Yale database: http://cvc.yale.edu/projects/yalefaces/yalefaces.html.

[8] J. Yang, D. Zhang, J.Y. Yang, Is ICA significantly better than PCA for face recognition? in: Proceedings of IEEE International Conference on Computer Vision, vol. 1, 2005, pp. 198-203.

[9] A.J. Bell, T.J. Sejnowski, The independent components of natural scenes are edge filters, Vision Res. 37 (23) (1997) 3327-3338.

\section{Authors}

M.Koteswara Rao is currently working as associate professor in ECE Department, QIS College of Engineering \& Technology, Ongole, A.P, India. He received his M.Tech from JNTUK, Kakinada. He has six years experience in the teaching undergraduate and post graduate students. His research interests in the area of content based image retrieval.

K.Veera Swamy is currently Professor in ECE department and Principal of QIS College of Engineering and Technology, Ongole, A.P, India. He received his Ph.D from JNTUK, Kakinada. He has fourteen years experience in teaching under graduate and post graduate students. His research interests are in the areas of image compression, image watermarking, Face recognition, CBIR, and networking protocols.
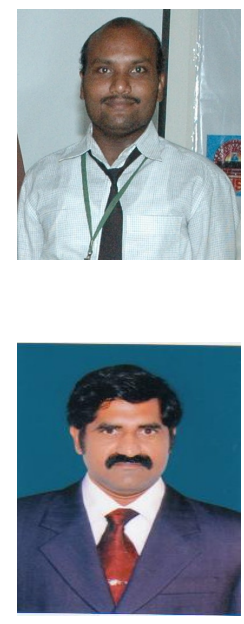

K.Anitha Sheela is currently working as Associate Professor in ECE Department, JNTUH College of Engineering, Hyderabad, A.P, India. She received her Ph.D from same Institute. She has done her B.Tech from REC Warangal, \& M.Tech from College of Engineering, Osmania University. She has Thirteen years experience in teaching under and post graduate students. Her research areas include Signal, Speech, Image processing and Neural networks.

B.Chandra Mohan is currently working as Professor \& HOD in ECE Department, Bapatla Engineering College, Bapatla, A.P, India. He received his Ph.D from JNTU College of Engineering, Kakinada, A.P, India; He has eighteen years experience in teaching under and post graduate students. His research interests are in the areas of Watermarking and communications.
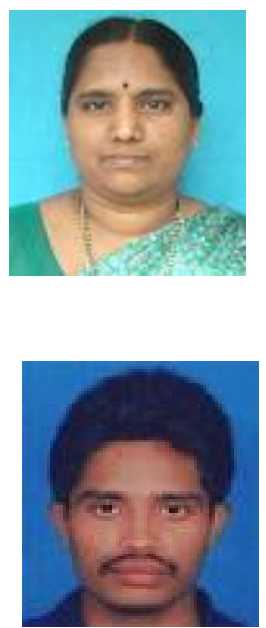